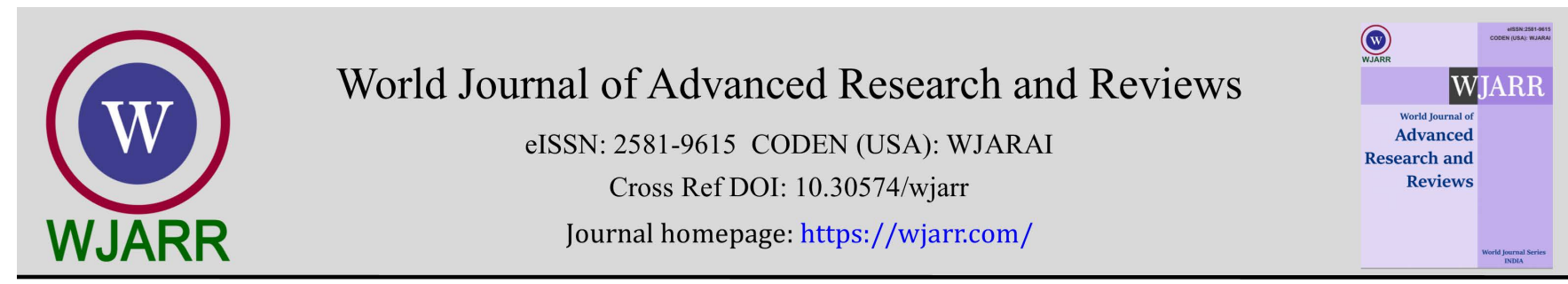

(REVIEW ARTICLE)

\title{
A review of genetic mapping of root depth in rice
}

\author{
Mohammad Sayedul Islam * \\ Department of Biochemistry and Molecular Biology, University of Chittagong, Chittagong-4331, Bangladesh.
}

World Journal of Advanced Research and Reviews, 2021, 10(03), 266-272

Publication history: Received on 13 May 2021; revised on 16 June 2021; accepted on 18 June 2021

Article DOI: https://doi.org/10.30574/wjarr.2021.10.3.0279

\begin{abstract}
Rice (Oryza sativa. L) is considered one of the most important cereal crops for human nutrition. It is required that rice production more than doubles to feed this growing population. To achieve that, better understanding of the root system will be required. It is very complex work to dissect specific genes which control the root structure variation due to the high adaptive plasticity of root development and the practical difficulty in phenotyping root traits and this represents a bottleneck for the efficient selection of specific root ideotypes. The main focus of this review is comparative evaluation of genetic mapping of rooting depth in rice based on QTL (Quantitative Trait Loci) study. Moreover, we will review the shortcomings and benefits of current genetic mapping procedure, as well as the future study to overcome these shortcomings.
\end{abstract}

Keywords: Rice; Root; Traits; Evaluation; QTLs; GWAS

\section{Introduction}

Rice (Oryza sativa) is a main staple crop around the world [1] and is one of the oldest domesticated crop species having fed more than 3.5 billion people than any other plant in human history [2]. Rice production should be increased more than $50 \%$ to meet the future demand by 2030 [3]. Root system plays a vital role in plant development and it is important to know the genetic and molecular mechanism controlling the root traits. Quantitative genetics is the study of the inheritance of traits that show a continuous distribution of phenotypes in a segregating population. In other words, quantitative genetics determines the continuous phenotypic variation of traits conditioned by allelic variation at several genomic loci, each with relatively small effect. Each genomic loci are called "quantitative traits loci (QTLs)" [4]. Each of the QTL segregates in a Mendelian fashion. Because of the relatively small effect of individual locus on the traits, the underlying genes are difficult to identify [5]. It is a really challenging task to detect the role of those each gene in the phenotypic expression. Most of the important agronomic traits, such as resistance to biotic and abiotic stress or yield are quantitative in nature and are controlled by several genes each of which has a small effect on phenotype.

\section{Genetic mapping and QTL}

Since the 1980's the advancement in molecular genetic markers has facilitated genetic dissection of complex traits in plants such as resistance to biotic and abiotic stress or yield through mapping quantitative trait loci (QTLs). Most of the natural variation that is observed in species including crop plants, including much that is of agronomic interest, is due to minor genetic differences in many genes [6]. The effect of most natural gene variants are small and produce small phenotypic effects which tend to be normally distributed, continuous and approximately additive [6]. QTL mapping aims to study and efficiently exploit this variation.

\footnotetext{
${ }^{*}$ Corresponding author: Mohammad Sayedul Islam

Department of Biochemistry and Molecular Biology, University of Chittagong, Chittagong-4331, Bangladesh.

Copyright (C) 2021 Author(s) retain the copyright of this article. This article is published under the terms of the Creative Commons Attribution Liscense 4.0.
} 
The QTL mapping approach involves a genome wide scan looking for statistical association between marker genotype and the trait values. Accurate phenotyping experiments on mapping populations in target environments and genetic linkage maps are therefore required to generate trait values and marker data for QTL analysis. The basic principle of QTL mapping was based on the concept that if the association between marker and trait value (QTL) is strong due to genetic linkage then they will segregate together thus the difference in those marker genotypes will be associated with different phenotypes [7]. But in the case of higher genetic distance between a marker and a QTL, there are likely to be number of recombination events and hence single marker analysis may not detect QTL at the relevant statistically significant threshold. An alternate to this is interval mapping (IM) analyses that estimates the position and effect of a QTL between pairs of flanking markers. This increases the power of detection of QTL effect and position. However it is biased when there are multiple QTLs [8]. Composite interval mapping overcome this problem by combining the interval mapping with multiple regression. The main use of the DNA marker is the construction of linkage map [9] and several steps involved to construct the map [10]. The first QTL mapping in a plant was performed in tomato which was reported by Paterson et al., [11]. From that time this approach has been widely used in the manipulation of breeding programme and genetic mapping of complex traits of many organism and crop plants even in the rice as well [12].

For marker assisted plant breeding (MAS) DNA markers which are tightly linked to agronomically important traits have been used as a very important molecular tool [13]. A great number of cereal researchers have utilized markers as a tool to identify main-effect genes, QTLs, or to introduce new characters in elite germplasm [14]. Recognizing the location of these genes and specific alleles offers the possibility to use MAS in cereals, because one of the major aims of plant breeding is the introgression of one or more favourable genes from a donor parent into the background of an elite variety.

\section{Mapping population}

In plant breeding programmes, QTL identification starts with a cross of two parents that ideally are both highly homozygous but are genetically divergent that causes them to display extreme phenotypes for the chosen traits [15]. Several different populations may be utilized for mapping within a given plant species. Progenies from the second filial generation $\left(\mathrm{F}_{2}\right)$, recombinant inbred lines (RILs), backcross (BC), double haploids (DHs), near isogenic lines (NILs), can be used for genetic mapping in self-pollinating species such as rice [16].

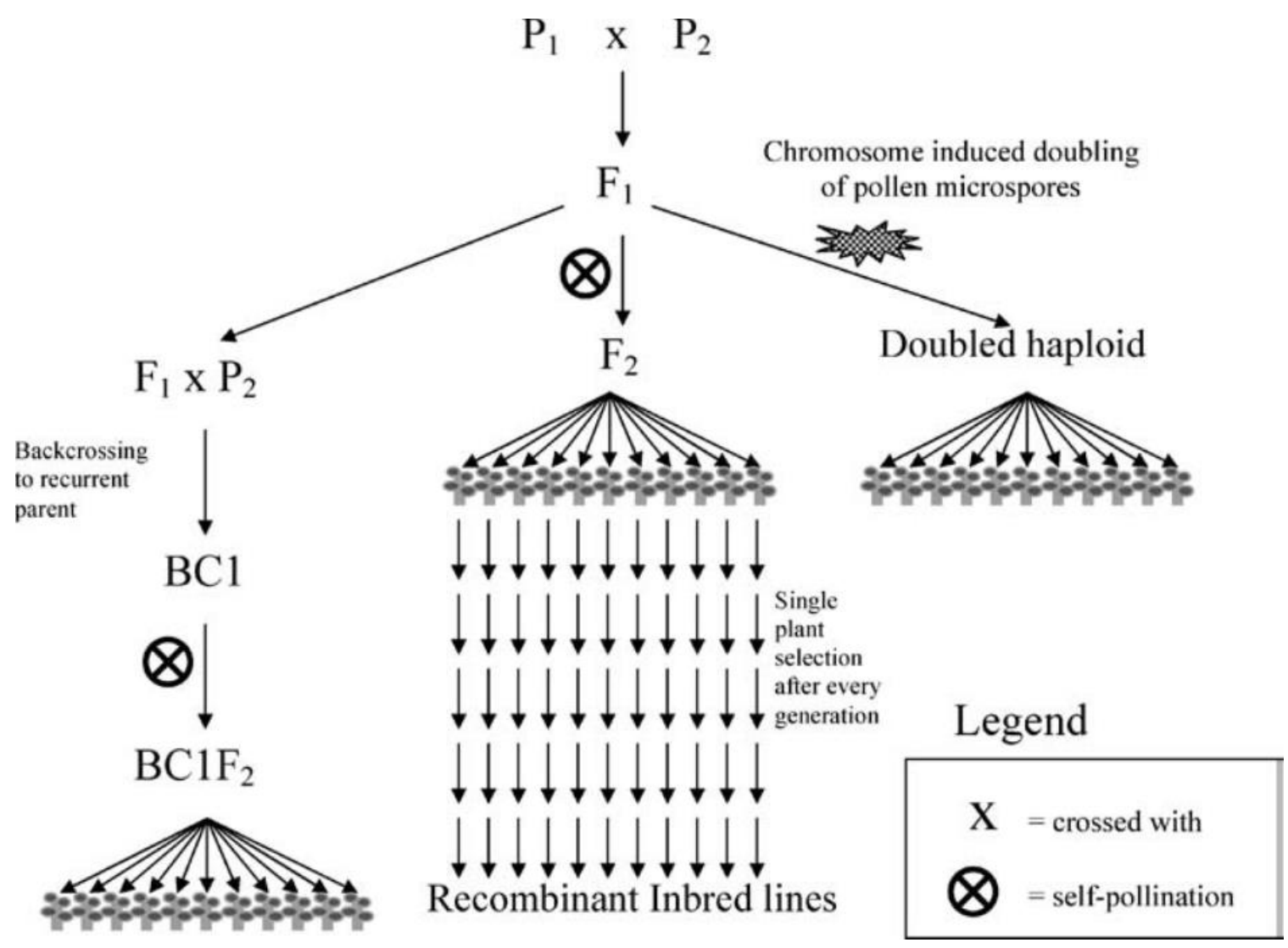

Figure 1 Main type of mapping populations for self-pollinating species [9] 
Populations of recombinant inbred lines (RILs) are usually developed from $\mathrm{F}_{2}$ individuals by the single seed descent method [17] and most genes are homozygous by six to eight generations [18]. Double haploid (DHs) population can be produced from $\mathrm{F}_{1} \mathrm{~s}$ through a spontaneous doubling of the chromosome complement of haploid microspores during in vitro culture. Early populations can be used to develop genetic maps such as $\mathrm{F}_{2}$ and $\mathrm{BC}_{1}$ [19]. However, they display one problem because of their short-lived duration and inability to maintain replicable individual lines. RIL and DH populations have big advantage for QTL analysis because these lines are stable permanent populations that can be distributed as seeds and can be tested repeatedly over years and locations and multiple traits [20]. Lack of heterozygosity is their major limitation by which the dominant gene effect cannot be detected.

Recently mapping populations such as Bala x Azucena recombinant inbred lines have been used to identify QTLs of various traits as like as drought tolerance [21], arsenic tolerance [22] and Striga hermonthica tolerance. This recombinant inbred population has been derived from the crosses between the shallow rooted Indica cultivar Bala and Azucena, a long and thick rooted Japonica, cultivar. This long and thick root characteristics extract water from the deep and Bala has better shoot mechanism which helps plants to avoid drought such as leaf rolling and greater osmotic adjustment [23].

\section{Molecular Markers and genetic linkage map}

There are several molecular markers other than RFLP, have been used for map construction such as microsatellites or simple sequence repeats (SSRs), expressed sequence tags (ESTs), cleaved amplified polymorphic sequence (CAPS), random amplified polymorphic DNA (RAPD), amplified fragment length polymorphism (AFLPs), inter simple sequence repeat (ISSR), diversity array technology (DArT) and single nucleotide polymorphism (SNP) [24]. Among all of the markers microsatellite or simple sequence repeats (SSR) are widely used, simple to generate, in addition to their being abundance in the genome, codominant and also exhibit variation in the number of motif repeats [25]. Because of this feature, SSRs are being widely used for the estimation of genetic diversity, establishment of varietal identity, construction of molecular genetic maps, assessment of hybrid purity and marker assisted backcross breeding [26]. Zhang et al.reported 52,000 micro satellite markers polymorphic between Indica and Japonica subpopulations [27].

Single nucleotide polymorphism (SNPs) is recently introduced and high throughput genetic markers. The value of SNPs is the number and the relatively low cost with which they can be produced and the ability to locate them on a reference genome.

An early demonstration of the power of SNPs in rice was the development of the Rice Diversity Panel 1 of 380 cultivars assessed for 36,000 polymorphic SNPs with an Affymetrix single nucleotide polymorphism (SNP) array (called the 44k SNP chip (containing 44,100 SNPs) [28]. High quality data (less than $4.5 \%$ missing data) has been produced by this SNP chip with $\sim 1$ SNP per $100 \mathrm{~kb}$ along the 12 chromosome. In the diversity panel was assessed for 34 traits linked to plant morphology, development grain quality and agronomic performance on field grown plants. This high-quality custom design 44k genotyping chip is now globally useful in rice genome wide association study for mapping QTL fore diverse complex traits [28].

A SNP dataset of around 3.6 million SNPs by low coverage sequencing 517 rice landraces has been produced by Bin Han group [29]. The study performed genome wide association study for 14 agronomic traits in the population of 0 . sativa subspecies Indica where QTL had been detected for all traits including heading date, drought tolerance, hull colour, tiller number, leaf angle, amylose content, grain width, grain length, grain weight, spikelet number.

\section{Association mapping}

Association mapping also called linkage disequilibrium (LD) mapping is a recent and powerful gene tagging mapping tool for detecting simple to complex quantitative traits in many crop species using the non-random associations of loci in haplotypes or random sample of a population [30]. The LD is described as the non-random form of association between alleles located at different loci within the genome. It is an unbiased association mapping approach for natural plant populations compared to the conventional linkage mapping. The principal is that if the marker is highly associated with the trait phenotype the marker is close to the underlying gene. That normally results in the identification of that QTL position with high accuracy (dependent on LD, the higher LD, lower the accuracy). Because, if the marker is too close to the gene, the association would have been removed by the cumulative historical recombination events [30]. The Linkage disequilibrium has been widely used to map and eventually clone a number of genes underlying the complex genetic traits in rice [31], barley [32] as well as in humans [33]. 
With the advances in genomic technology and improvements in powerful statistical methods, exploiting natural diversity using association mapping has become an achievable and low-cost tool for plant investigation projects. Advantages include many alleles evaluated simultaneously, higher resolution mapping because of the utilization of many recombination events from a large amount of meiosis throughout the germplasm development history, decreased research time since there is no need to develop a bi-parental population.

\subsection{Linkage disequilibrium (LD) and population structure}

Diverse panel of Oryza sativa are reported to have similar or slightly elevated levels of LD compared to species such as Arabidopsis, maize and humans. The average extent of LD in rice has been estimated at between 50-500 kb [34], depending on the germplasm evaluated, compared to 10-250 kb in Arbidopsis and human [35], 100-500 kb in commercial elite maize inbred and 1-2 kb diverse maize. The inbreeding nature of Oryza sativa coupled with its demographic history, are major determinants of genome wide pattern of LD. Strong selective pressure over the course of rice domestication has also lead to deep population substructure ( $F_{\text {st }}=0.23$ to 0.57) [36], which sets it apart from Arabidopsois, in which population structure is gradual across geographic [37]. Population structure can lead to false positives in association mapping studies, and must be taken into account [37]. The mixed model has been demonstrated to work well in both maize and Arabidopsis [37] and it has also shown its ability to greatly reduce the false positive rates in rice when used within a single subpopulation, though it may introduce false negatives when used on a diversity panel representing all domesticated subpopulations [28].

\subsection{QTL studies for root traits in rice}

In recent years, in rice there are many QTL analysis for root morphological characteristics such as root length and thickness have already been reported in different mapping populations and Courtois et al, reported a meta-analysis of 675 detected root QTLs including 103 QTLs for maximum root length across 12 chromosomes, while 89 QTLs for root systems architecture traits in gel base image analysis were detected by Topp et al. [39]. A meta-analysis of drought related QTL in the Bala x Azucena mapping population study was performed by Khowaja et al.,[40]. A number of QTLs was identified in five trait categories drought avoidance, plant height, plant biomass, leaf morphology and root traits by 13 experiments in 25 individual screens in this population. It included 505 previously identified root trait QTLs.

\section{Comparing the mapping methods in rice}

In recent years many studies have been performed to detect quantitative trait loci (QTLs) for root traits in rice. Among these root morphological traits such as root thickness, maximum root length, volume and distribution have been performed in different mapping populations [41]. Large numbers of QTLs for root traits have been identified. For example, Topp et al., detected 89 QTLs for root systems architecture traits while 103 QTLs for maximum root length [39] where detected across the 12 chromosomes of rice [40]. From the identification of QTLs, it is possible to identify either smaller genomic regions responsible for the genetic variation suitable for molecular breeding or the gene responsible for the variation. For example, the major QTL qRL 6.1 for root length on chromosome 6 mapped by Obara et al. [42] has been narrowed down to a candidate genomic region of 337kb. In addition, a major QTL for deep rooting has been cloned and the gene identified as Dro1 [43].

A high-quality reference genome sequence, self-fertilization and phenotypic resources make rice an ideal crop to use genome wide association studies (IRGSP, 2005). Due to a relatively small linkage disequilibrium, the main advantage of GWAS (Genome Wide Association Study). over conventional QTL mapping is the smaller number of positional candidate genes that underlie detected QTLs. Jin et al. suggests that level and distribution of linkage disequilibrium and population structure are two of the things that are important in the success of a GWAS along with marker density, the degree of phenotype variation and the accuracy with which it can be measured [44]. To date, GWAS has already been used to identify the genomic regions associated with flowering time, grain element composition, panicles per plant, seed number per panicle, seed morphology, blast resistance, amylose content and protein content traits using the rice accessions called Rice Diversity Panel 1(RDP1) which is 421 accessions with 36,901 high quality SNPs [22] [23] [28]. GWAS mapping was also performed by Huang et al. who used 517 accessions with 3.6 million SNPs. Tiller number grain morphology, amylose content, heading date and drought tolerance traits were studied [32]. They detected 32 loci for flowering time and 10 grain related traits. GWAS mapping has been used to identify the loci controlling the root traits in rice panel. 


\section{Conclusion}

In this study we evaluate a number of genetic mapping methods which demonstrated the quantitative traits loci (QTLs) for root traits. Using these mapping methods positional candidate genes that underlie the detected QTLs could be achieved. Though some of the mapping methods have some limitations they are very useful tools to identify the genes controlling the root traits.

\section{Compliance with ethical standards}

\section{Acknowledgments}

Thanks to Research and publication cell and Department of Biochemistry \& Molecular Biology, University of Chittagong, Bangladesh for providing supporting documents.

\section{Disclosure of Conflict of Interest}

The author declares no conflicts of interest regarding the publication of this article.

\section{References}

[1] Fuller DQ, Sato YI, Castillo C, Qin L, Weisskopf AR, Kingwell-Banham EJ, Song J, Ahn SM, Van Etten J. Consilience of genetics and archaeobotany in the entangled history of rice. Archaeological and Anthropological Sciences. $2010 ; 2(2): 115-131$.

[2] Caicedo AL, Williamson SH, Hernandez RD, Boyko A, Fledel-Alon A, York TL, Polato NR, Olsen KM, Nielsen R, McCouch SR, Bustamante CD. Genome-wide patterns of nucleotide polymorphism in domesticated rice. PLoS Genet. 2007; 3(9): 1745-1756.

[3] Ahmadi N, Audebert A, Bennett MJ, Bishopp A, de Oliveira AC, Courtois B, Diedhiou A, Diévart A, Gantet P, Ghesquière A, Guiderdoni E. 2014. The roots of future rice harvests. Rice. 2014; 7(1): 1-9.

[4] Tanksley SD, Nelson JC. Advanced backcross QTL analysis: a method for the simultaneous discovery and transfer of valuable QTLs from unadapted germplasm into elite breeding lines. Theoretical and Applied Genetics. 1996; 92(2): 191-203.

[5] Yano M, Harushima Y, Nagamura Y, Kurata N, Minobe Y, Sasaki T. Identification of quantitative trait loci controlling heading date in rice using a high-density linkage map. Theoretical and Applied Genetics. 1997; 95(7): 1025-1032.

[6] Kearsey MJ, Farquhar AGL. QTL analysis in plants; where are we now?. Heredity. 1998; 80(2): 137-142.

[7] Kearsey MJ. The principles of QTL analysis (a minimal mathematics approach). Journal of experimental botany. 1998; 49(327): 1619-1623.

[8] Lander ES, Green P, Abrahamson J, Barlow A, Daly MJ, Lincoln SE, Newburg L. MAPMAKER: an interactive computer package for constructing primary genetic linkage maps of experimental and natural populations. Genomics. 1987; 1(2): 174-181.

[9] Collard BC, Jahufer MZZ, Brouwer JB, Pang ECK. An introduction to markers, quantitative trait loci (QTL) mapping and marker-assisted selection for crop improvement: the basic concepts. Euphytica. 2005; 142(1): 169-196.

[10] Han JW, Zheng HF, Cui Y, Sun LD, Ye DQ, Hu Z, Zhang XJ. Genome-wide association study in a Chinese Han population identifies nine new susceptibility loci for systemic lupus erythematosus. Nature genetics. 2009; 41(11): 1234-1237.

[11] Paterson AH, Lander ES, Hewitt JD, Peterson S, Lincoln SE, Tanksley SD. Resolution of quantitative traits into Mendelian factors by using a complete linkage map of restriction fragment length polymorphisms. Nature. 1988; 335(6192): 721-726.

[12] Yano M, Sasaki T. Genetic and molecular dissection of quantitative traits in rice. Oryza: from molecule to plant. 1997; 145-153. 
[13] Narshimulu G, Jamaloddin M, Vemireddy LR, Anuradha G, Siddiq E. Potentiality of evenly distributed hypervariable microsatellite markers in marker-assisted breeding of rice. Plant Breeding. 2011; 130(3): 314320 .

[14] Moose SP, Mumm RH. Molecular plant breeding as the foundation for 21st century crop improvement. Plant physiology. 2008; 147(3): 969-977.

[15] Wu P, Liao CY, Hu B, Yi KK, Jin WZ, Ni JJ, He C. QTLs and epistasis for aluminum tolerance in rice (Oryza sativa L.) at different seedling stages. Theoretical and Applied Genetics. 2000; 100(8), 1295-1303.

[16] Doerge RW. Mapping and analysis of quantitative trait loci in experimental populations. Nature Reviews Genetics. 2002; 3(1): 43-52.

[17] Price AH, Steele KA, Moore BJ, Barraclough PP, Clark LJ. A combined RFLP and AFLP linkage map of upland rice (Oryza sativa L.) used to identify QTLs for root-penetration ability. Theoretical and Applied Genetics. $2000 ; 100(1): 49-56$.

[18] Mackill DJ, Ismail AM, Pamplona AM, Sanchez DL, Carandang JJ, Septiningsih EM. Stress tolerant rice varieties for adaptation to a changing climate. Crop, Environment \& Bioinformatics. 2010; 7: 250-259.

[19] Price AH, Tomos AD. Genetic dissection of root growth in rice (Oryza sativa L.). II: mapping quantitative trait loci using molecular markers. Theoretical and Applied Genetics. 1997; 95(1-2): 143-152.

[20] Yano M, Sasaki T. Genetic and molecular dissection of quantitative traits in rice. Oryza: from molecule to plant. 1997; 145-153.

[21] Price AH, Steele KA, Moore BJ, Jones RGW. Upland rice grown in soil-filled chambers and exposed to contrasting water-deficit regimes: II. Mapping quantitative trait loci for root morphology and distribution. Field crops research. 2002; 76(1): 25-43.

[22] Norton GJ, Nigar M, Williams PN, Dasgupta T, Meharg AA, Price AH. Rice-arsenate interactions in hydroponics: a three-gene model for tolerance. Journal of experimental botany. 2008; 59(8): 2277-2284.

[23] Price AH, Cairns JE, Horton P, Jones HG, Griffiths H. Linking drought-resistance mechanisms to drought avoidance in upland rice using a QTL approach: progress and new opportunities to integrate stomatal and mesophyll responses. Journal of experimental botany. 2002; 53(371): 989-1004.

[24] Rafiq M, Liaqat S, Ahmed RI, Najeebullah M, Ahmad RT, Karim A, Jabbar A. An overview of marker assisted selection and QTL mapping in cotton. International Journal of Agronomy and Agricultural Research (IJAAR). 2016; 8(1): 71-80.

[25] Narshimulu G, Jamaloddin M, Vemireddy LR, Anuradha G, Siddiq E. Potentiality of evenly distributed hypervariable microsatellite markers in marker-assisted breeding of rice. Plant Breeding. 2011; 130(3): 314320.

[26] Sundaram RM, Vishnupriya MR, Biradar SK, Laha GS, Reddy GA, Rani NS, Sonti RV. Marker assisted introgression of bacterial blight resistance in Samba Mahsuri, an elite indica rice variety. Euphytica. 2008; 160(3): $411-422$.

[27] Zhang Z, Deng Y, Tan J, Hu S, Yu J, Xue Q. A genome-wide microsatellite polymorphism database for the indica and japonica rice. DNA research. 2007; 14(1): 37-45.

[28] Zhao K, Tung CW, Eizenga GC, Wright MH, Ali ML, Price AH, McCouch SR. Genome-wide association mapping reveals a rich genetic architecture of complex traits in Oryza sativa. Nature communications. 2011; 2(1): 1-10.

[29] Huang X, Sang T, Zhao Q, Feng Q, Zhao Y, Li C, Han B. Genome-wide association studies of 14 agronomic traits in rice landraces. Nature genetics. 2010; 42(11): 961.

[30] Chang C, Hu Y, Sun S, Zhu Y, Ma G, Xu G. Proton pump OsA8 is linked to phosphorus uptake and translocation in rice. Journal of experimental botany. 2009; 60(2): 557-565.

[31] Famoso AN, Zhao K, Clark RT, Tung CW, Wright MH, Bustamante C, McCouch SR. Genetic architecture of aluminum tolerance in rice (Oryza sativa) determined through genome-wide association analysis and QTL mapping. PLoS Genet. 2011; 7(8): e1002221.

[32] Kraakman AT, Niks RE, Van den Berg PM, Stam P, Van Eeuwijk FA. Linkage disequilibrium mapping of yield and yield stability in modern spring barley cultivars. Genetics. 2004; 168(1): 435-446. 
[33] Taniguchi H, Lowe CE, Cooper JD, Smyth DJ, Bailey R, Nutland S, Todd JA. Discovery, linkage disequilibrium and association analyses of polymorphisms of the immune complement inhibitor, decay-accelerating factor gene (DAF/CD55) in type 1 diabetes. BMC genetics. 2006; 7(1): 1-9.

[34] McNally KL, Childs KL, Bohnert R, Davidson RM, Zhao K, Ulat VJ, Leach JE. Genomewide SNP variation reveals relationships among landraces and modern varieties of rice. Proceedings of the National Academy of Sciences. 2009; 106(30): 12273-12278.

[35] Nordborg M, Borevitz JO, Bergelson J, Berry CC, Chory J, Hagenblad J, Weigel D. The extent of linkage disequilibrium in Arabidopsis thaliana. Nature genetics. 2002; 30(2): 190-193.

[36] Garris AJ, Tai TH, Coburn J, Kresovich S, McCOUCH S. Genetic structure and diversity in Oryza sativa L. Genetics. 2005; 169(3): 1631-1638.

[37] Atwell S, Huang YS, Vilhjálmsson BJ, Willems G, Horton M, Li Y, Meng D, Platt A, Tarone AM, Hu TT, Jiang R. Genome-wide association study of 107 phenotypes in Arabidopsis thaliana inbred lines. Nature. 2010; 465(7298): 627-631.

[38] Courtois B, Ahmadi N, Khowaja F, Price AH, Rami JF, Frouin J, Ruiz M. Rice root genetic architecture: meta-analysis from a drought QTL database. Rice. 2009; 2(2): 115-128.

[39] Topp CN, Iyer-Pascuzzi AS, Anderson JT, Lee CR, Zurek PR, Symonova O, Benfey PN. 3D phenotyping and quantitative trait locus mapping identify core regions of the rice genome controlling root architecture. Proceedings of the National Academy of Sciences. 2013; 110(18): E1695-E1704.

[40] Khowaja FS, Norton GJ, Courtois B, Price AH. Improved resolution in the position of drought-related QTLs in a single mapping population of rice by meta-analysis. BMC genomics. 2009; 10(1): 1-14.

[41] Price AH, Cairns JE, Horton P, Jones HG, Griffiths H. Linking drought-resistance mechanisms to drought avoidance in upland rice using a QTL approach: progress and new opportunities to integrate stomatal and mesophyll responses. Journal of experimental botany. 2002; 53(371): 989-1004.

[42] Obara M, Tamura W, Ebitani T, Yano M, Sato T, Yamaya T. Fine-mapping of qRL6. 1, a major QTL for root length of rice seedlings grown under a wide range of $\mathrm{NH} \mathrm{4+} \mathrm{concentrations} \mathrm{in} \mathrm{hydroponic} \mathrm{conditions.} \mathrm{Theoretical} \mathrm{and}$ Applied Genetics. 2010; 121(3): 535-547.

[43] Uga Y, Okuno K, Yano M. Dro1, a major QTL involved in deep rooting of rice under upland field conditions. Journal of experimental botany. 2011; 62(8): 2485-2494.

[44] Jin L, Lu Y, Xiao P, Sun M, Corke H, Bao J. Genetic diversity and population structure of a diverse set of rice germplasm for association mapping. Theoretical and Applied Genetics. 2010; 121(3): 475-487. 